\title{
Cache Management Based on Node Status in Delay Tolerant Networks
}

\author{
Jun-She Wang \\ School of Information Science and Engineering, Hebei \\ University of Science and Technology \\ Shijiazhuang, Hebei, China \\ wangjunshe@hebust.edu.cn
}

\begin{abstract}
Delay Tolerant Network (DTN) is a kind of wireless network with limited resources and often occurs congestion. The effective cache management can make full use of the network resources and reduce the network congestion. Therefore, aiming at the deficiency of the current research of cache management, this paper puts forward a kind of cache management strategy based on node status. With the knowledge of statistical analysis and control, the message queue and transmission rate are managed dynamically by detecting node activity and load. The experimental results show that, under the cache management, the network congestion was obviously reduced, and the utilization rate of resources has been improved, and the communication quality of the DTN is guaranteed.
\end{abstract}

Keywords-node status; interval time; queue management; load control;

\section{INTRODUCTION}

DTN is an abstract network model, which is used to solve the communication problem of the wireless network. As a result of the node mobility and limited resources, DTN cannot guarantee a long-term and complete end-toend path between the source nodes to the destination node. DTN has the characteristics of long latency and easy interrupt [1].

The routing strategy that determines the end-to-end path in traditional network is no longer applicable to the DTN, so DTN has its own unique routing strategy [2]. In order to make full use of the mobility of nodes, Most of the DTN routing algorithms use the means of message replication.

DTN uses a storage - carry - forward transmission mechanism [3], so each node needs to be responsible for keeping and carrying messages over a long period of time until the messages is delivered. Because the frequent interruption of the link, long term message storage and excessive replication have brought great burden to the transmission, and it is extremely easy to cause the network

\author{
Bin Zhang \\ School of Information Science and Engineering, Hebei \\ University of Science and Technology \\ Shijiazhuang, Hebei, China \\ 770961827@qq.com
}

congestion [4]. Therefore, cache management is of great significance to control the congestion of DTN.

\section{RELATED RESEARCH}

The cache management of DTN is mainly aimed at the management of the node's cached data and message queue $[5,6]$. There are two directions for research on cache management at present: one is based on the routing algorithm [7]. By assigning a fixed number of message copies to the source node, Spray and Wait routing improves the diffusion of the message [7]. In addition, Prophet routing algorithm uses the delivery probability to determine which messages are retained or prioritized to be sent [8]. On the basis of the former, Max Prophet strategy not only sorts the message by encounter probability, but also restrains the transmission of the low priority message [9]. These algorithms can avoid the congestion caused by long term retention of message to some extent, but their scope of application and development is poor.

The other strategies is not dependent on the routing algorithm. Some strategies selectively discard messages according to the message properties such as the survival time (Drop First) and the location in the queue (Drop Tail), thereby maintaining the storage capacity of the node [10, 11]. The core idea of pre distribution and quota control method is to adjust and optimize the performance by measuring the global network state, and then ease the network congestion [12]. For example, by detecting the number and the density of nodes, the number of message copies set in advance. These algorithms are suitable for a wide range of application, but lack of real-time and initiative, which affect the performance of congestion control.

To sum up, in view of the shortcomings of previous cache management strategies in real time and adaptability, this paper proposes an adaptive cache management strategy. The aim of this strategy is to improve the network performance by managing node caching more flexibly.

\section{Cache Management Strategy}

The overall framework of the cache management strategy based on node status (CMBNS) is shown in Fig. 1. 


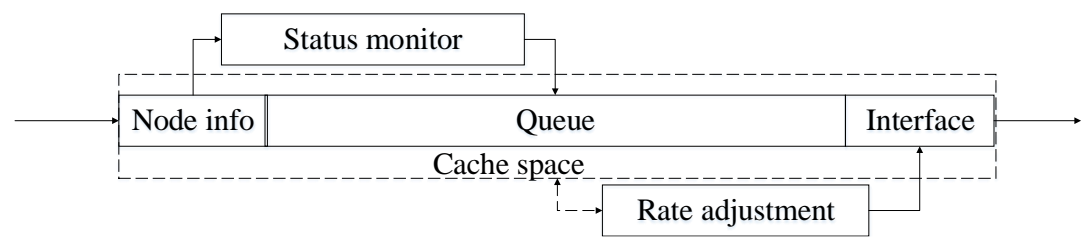

Figure 1. The overall framework of CMBNS

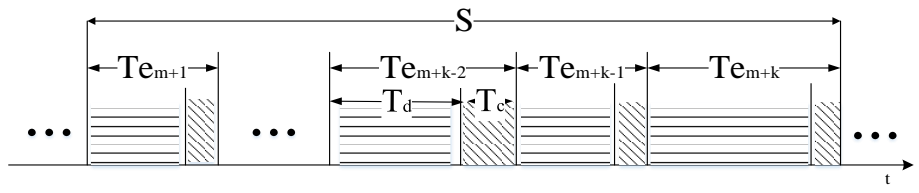

Figure 2. Inspection period of nodes

This strategy mainly includes two aspects:

1) Multi queue sequencing. Combined with the historical information of the interval time of the node meeting, the node's activity is quantified, and then the realtime multi queue sequencing of the nodes with different active degree is carried out, so as to ensure the maximum transmission utility of each node.

2) Rate control. According to the condition of node load, the feedback mechanism is used to control the transmission rate to ensure the load balance.

\section{A. Node Activity Prediction}

Calculate the node activity, the first to determine the node's state-cycle.

The time that a node passes from one meeting to next is a encounter period $T_{e}$, which includes the Interval time $T_{d}$ before the meeting the Contact time $T_{c}$ after the meeting.

Analyzing the mobility of nodes, the interval time reflects the frequent degree that a node encounters with other nodes, which is the activity of nodes. Define $\bar{I}$ as the average interval time. The formula of node vitality is as follows:

$$
V=1 / \bar{I}
$$

Frequent detection of node activity will seriously affect the network performance, so select the previous $k$ cycles containing the current $T_{e}$ as an Inspection period $S$.

The average interval of the next inspection cycle is predicted by the exponential smoothing method. The formula is as follows:

$$
\overline{I_{t+1}}=\alpha I_{t}+(1-\alpha) \overline{I_{t}}
$$

$\overline{I_{t+1}}$ is the smoothing value of average interval time for the current inspection period $S_{t}$, which is also the average interval time prediction value for the next $S_{t+1}$. $I_{t}$ is the actual average interval time of the current $S_{t}$, and $\bar{I}_{t}$ is the predictive value of the current $S_{t}$. In reality, the movement of DTN node has certain regularity, so we need to use the exponential smoothing predictive value for second times. The calculation formula of the final interval time $\overline{I_{t+1}}$ prediction value is as follows:

$$
{\overline{I_{t+1}}}^{\prime}=\alpha \overline{I_{t+1}}+(1-\alpha) \overline{I_{t}}
$$

Where $\bar{I}_{t}$ is the predicted value of the two exponential smoothing method for the current $S_{t}$, and $\alpha$ is the smoothing factor.

\section{B. The Partition of Message Priority}

Without considering the message content, the main indicators of the impact of the quality of the message in DTN are the survival time and the number of copies of the message. Therefore, it is necessary to queue messages through these indicators.

Define $T T L$ as the survival time of the message. It is clear that the priority of the message's survival time is higher than the number of copies of the message.

Therefore, according to the active degree of nodes, the message is sorted by multiple queues. The first step, according to the relationship between the node's vitality and message's $T T L$, the messages is divided into multiple queues. The second step is to estimate the utility of messages in different queues and queue them up. The framework of multi queue management is show in Fig. 3. 


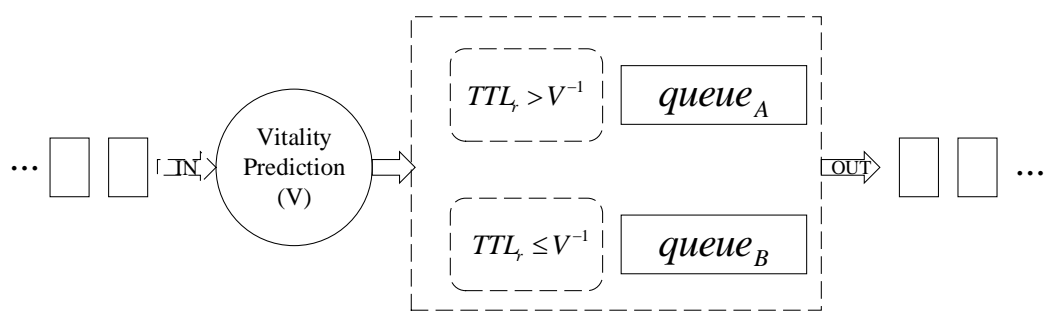

Figure 3. The framework of multi queue management

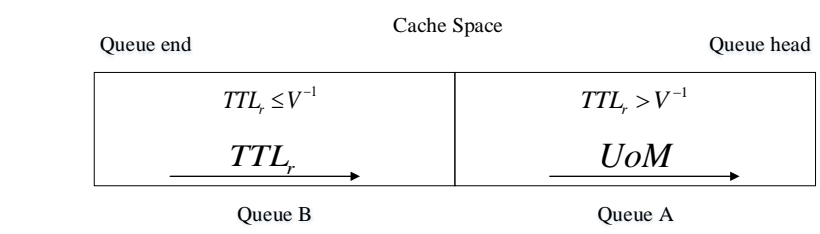

Low priority

High priority

Figure 4. Fig. 4. The diagram of message sequencing

$V$ indicates that the node's active degree is inversely proportional to its average encounter interval time $\bar{I}$.TTL $L_{r}$ is the residual survival time of message.

1) When $T T L_{r} \leq V^{-1}$, the message in the $\bar{I}$ will be deleted, the message is the possibility of successful transmission is low, into the queue $\mathrm{B}$.

2) When $T T L_{r}>V^{-1}$, the message can survive for a long time, the node can give full play to the advantages of custody transfer. The possibility and extent to message transmission in the network are higher, should be placed in the queue $\mathrm{A}$.

The message's $T T L_{r}$ is short in queue $\mathrm{B}$, needed to be sorted by $T T L_{r}$, Because the message's $T T L_{r}$ is longer, its transmission is bigger. Message in queue A has a high transmission value, so it needs to consider the effect of message copy on message utility. The diagram of message sequencing is s shown in Fig. 4.

$U o M$ in Fig. 4 is the utility value of a single message, which is affected by the number of message copies and the active degree of the node, so it is necessary to grade the node's activity level. The active level of the node is defined as $W=\left\{w_{1}, w_{2}, \cdots, w_{\text {med }}, \cdots, w_{2 \text { med }-1}\right\}$. When the node has a moderate active degree $V_{\text {med }}$, it has a moderate level of activity $W_{\text {med }}$. So the formula for calculating the active level of node $i$ is as follows:

$$
W_{i}=w_{\text {med }}+\left\lceil\left(V_{i}-V_{\text {med }}\right) / \Delta V\right\rceil
$$

When the average interval time of the node is the average interval time of the network, that is, $\bar{I}=\overline{T_{d}}$, the node has a moderate active degree $V_{\text {med }}$. Therefore, it is necessary to estimate the $\overline{T_{d}}$. The average transmission delay of the direct transmission is calculated as follows when the node's range of motion is $\sqrt{N} * \sqrt{N}$ :

$$
\overline{D T}=(c N \log N) / 2
$$

Assuming that the number of copies of a message is $n-1, n$ is the total number of nodes in the network. That is, all nodes except the destination node are carrying a copy of the message. For each copy of the transmission is a direct transmission, so the average interval time of encounter between any two nodes is calculated as follows:

$$
\overline{T_{d}}=\overline{D T}\left(\frac{1}{n-1}+\frac{1}{n-2}\right)=\frac{(2 n-3) c N \log N}{2(n-1)(n-2)}
$$

The active level of the node as an active weight of a copy, so the total value of the message is defined as the sum of the active weights of all message copies, the formula is as follows:

$$
U=\sum_{m} W
$$

As mentioned above, the total value of the message not only reflect the number of message copies, but also reflect the extent of the spread of the message. The greater the spread of the message, the smaller the value of a single copy, so the utility value of the message $m$ in node $i$ is defined as follows:

$$
U o M_{i}=W_{i} / \sum_{m} W
$$

When the utility value is large, the diffusion degree of the message is small, and the message's quality is high. Therefore, the messages are sorted by the high-to-low utility value in queue $\mathrm{A}$. 


\section{The Rate Adjustment under Load Detection}

In order to balance the load and improve the resource utilization, the transmission rate of the node is needed to be adjusted with a limited cache space. The load status of the node can be judged according the changes of cache occupancy. And then the transmission rate is adjusted by the dynamic threshold. The dynamic load threshold calculation formula is as follows:

$$
T(t)=\alpha\left(B-B_{f}(t)\right)
$$

$B$ is the node's own cache space capacity , $B_{f}(t)$ is the cache occupancy, and $\alpha$ is an adjustment factor. The relationship between $T(t)$ and $B_{f}(t)$ is shown in Fig. 5.

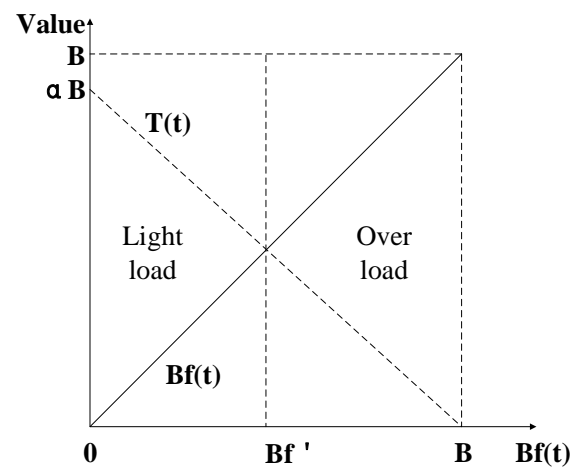

Figure 5. The relationship of load threshold and cached data

It is obvious that the load threshold $T$ decreases with the decrease of the remaining space of the cache. That is, $B_{f}$ and $T$ are negatively correlated. In order to ensure that the node is in a relatively stable state of the load, the transmission rate is adjusted through the negative feedback mechanism.

Obviously, when the two curves intersect, the node's cache state is the best. At this time the transmission rate $v_{c}$ is the initial value $v_{\text {init }}$.

When the node's cache size is changes, its transmission rate is adjusted adaptively.

When $B_{f}>T$, the node is in overload condition. It is more likely to be congested, and it's transmission rate need to be improved:

$$
v_{c}=v_{\text {init }}+\beta\left(B_{f} / T\right)
$$

When $B_{f} \leq T$, the node is idle. It is less likely to be congested, so it's transmission rate can be reduced appropriately:

$$
v_{c}=v_{\text {init }}-\beta\left(T / B_{f}\right)
$$

The above formula is in the range of $\left(v_{\text {init }}-\alpha \beta B, v_{\text {init }}+\beta B\right)$. If $v_{c} \in\left(v_{\min }, v_{\max }\right)$, then $\beta \in\left(0, B^{-1} * \min \left(\alpha^{-1}\left(v_{\text {init }}-v_{\min }\right), \alpha\left(v_{\max }-v_{\text {init }}\right)\right)\right)$.

\section{EXPERIMENTAL VERIFICATION}

\section{A. Experimental Environment}

In order to simulate the DTN network with dynamic characteristics of network topology, the ONE software is used as the simulation tool.

The configuration of the simulation scene is shown in Table 1.

\section{B. Experimental Analysis}

The performance of CMBNS is verified by comparing with Drop Front strategy (DF) and Max Prophet strategy (MaxProp) in three aspects: delivery probability, transmission delay and queue length.

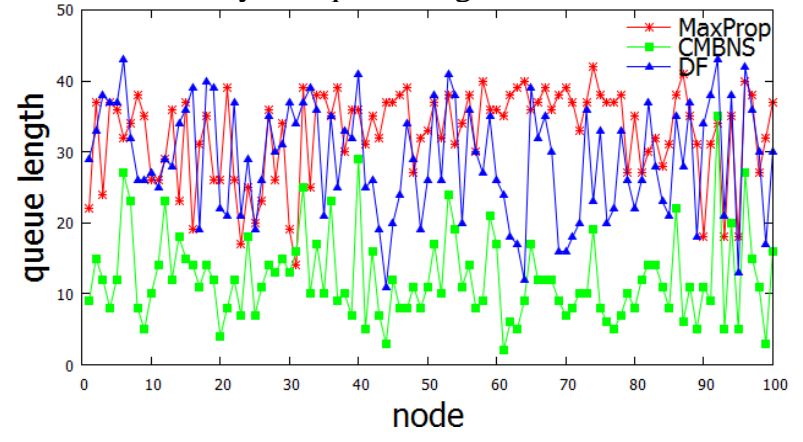

Figure 6. The queue length of each node

TABLE 1 T. THE SELECTION OF SIMULATION PARAMETERS

\begin{tabular}{cccc}
\hline Simulation parameters & & Value & Trams \\
\hline Types of nodes & Pedestrians & Cars & 6 \\
Number of nodes & 80 & 40 & $7 \sim 10$ \\
Node speed (m/s) & $0.5 \sim 1.5$ & $2.7 \sim 13.9$ & $7 \sim 10$ \\
Node stagnation time (s) & $0 \sim 120$ & $0 \sim 120$ & $10 / 1000$ \\
Communication range (m) & 10 & 10 & Map Route \\
Moving model & Shortest Path & Shortest Path based on Map & \\
Message size (Bytes) & & $(500 \mathrm{k}, 1 \mathrm{M})$ & $4500 \times 3400$ \\
Network range $\left(\mathrm{m}^{2}\right)$ & & & \\
\hline
\end{tabular}




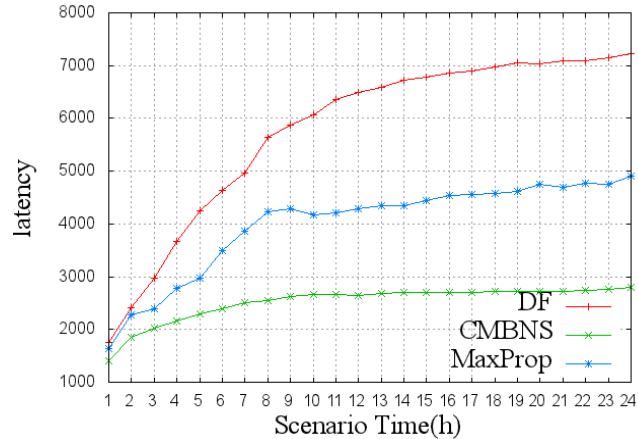

Figure $7 . \quad$ Transmission latency

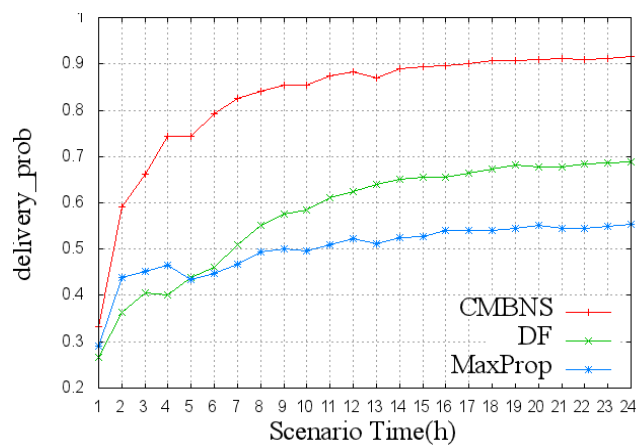

Figure $8 . \quad$ The delivery probability

As shown in Fig. 6, compared to DF and MaxProp, the queue length of CMBNS is the least, that is, the cache utilization is the highest. The reason is that CMBNS estimate the message's utility in the nodes with different active degree, which ensure the maximum utility of the sort and the timely transmission of message. In addition, the transmission rate is adjusted in a timely manner through the load detection of nodes, which improves the stability of the resource utilization, so the queue length is more stable and the load is more balanced. The corresponding transmission delay has also been a substantial decline, as shown in Fig. 7. Fig. 8 reflects the change of delivery probability under three different cache management strategies. With the gradual stabilization of the system, CMBNS has a higher delivery probability. Compared to the other two strategies, the nodes in CMBNS will give priority to transfer of high utility message. These messages rarely stranded in the network, and have strong communication ability.

\section{CONCLUSIONS}

This paper analysis the congestion of DTN and proposes the CMSBNS. On the one hand, the real-time multi queue sequencing of the nodes with different active degree is cached. on the other hand, the transmission rate of nodes is dynamically controlled according to the load conditions of the nodes. The simulation results show that CMBNS through real-time cache management to ensure the rationality of the message queue, and limit the transmission efficiency of the network, so as to effectively reduce the transmission delay, improve the delivery probability, better balance the load and prevent congestion.

\section{REFERENCE}

[1]. Fall, K.R.: A Delay-Tolerant Network Architecture for Challenged Internets. ACM SIGCOMM Computer Communication Review. 33(4), 27--34 (2003)

[2]. Cao, Y., Sun, Z.: Routing in Delay/Disruption Tolerant Networks: A Taxonomy, Survey and Challenges. IEEE Communications Surveys \& Tutorials. 15(2), 654--677 (2013)

[3]. Yu, Q., Sun, X., Wang, R., et al.: The effect of DTN custody transfer in deep-space communications. IEEE Wireless Communications. 20(5), 169--176 (2013)

[4]. Silva, A.P., Hirata, C.M., Burleigh, S., et al.: A survey on congestion control for delay and disruption tolerant networks. Ad Hoc Networks. 25, 480--494 (2015)

[5]. Hoseong, L.: Combined Optimal Control of Activation and Transmission in Delay-Tolerant Networks. IEEE/ACM Transactions on Networking. 21(2), 482-494 (2013)

[6]. Jain, S., Chawla, M.: Survey of buffer management policies for delay tolerant networks. Journal of Engineering. 1--7 (2014)

[7]. Spyropoulos, T., Psounis, K., Raghavendra, C.S.: Spray and wait: an efficient routing scheme for intermittently connected mobile networks. ACM SIGCOMM Workshop on Delay-Tolerant Networking. 252-259 (2005)

[8]. Lindgren, A., DORIA, A., DAVIES, E., et al.: Probabilistic Routing Protocol for Intermittently Connected Networks. LETF RFC 6693 (2012)

[9]. Burgess, J., Gallagher, B., Jensen, D., et al.: MaxProp: Routing for Vehicle-Based Disruption-Tolerant Networks. 25th IEEE International Conference on Computer Communications, pp. 1--11. IEEE Press, Barcelona (2006)

[10]. Wei, K., Guo, S., Zeng, D., et al.: A multi-attribute decision making approach to congestion control in delay tolerant networks. 14th IEEE International Conference on Communications. pp. 2742-2747. IEEE Press, Australia (2014)

[11]. Farkiani, B., Sabaei, M.: A novel buffer management policy based on prediction in Delay Tolerant Networks. 7th IEEE International Symposium on Telecommunications, pp. 27-34. IEEE Press, Iran (2014)

[12]. Lo, S.C., Tsai, C.C., Lai, Y.H.: Quota-control routing in delaytolerant networks. Ad Hoc Networks. 25(PB), 393--405 (2015) 